\title{
The effect of the ion beam energy on the properties of indium tin oxide thin films prepared by ion beam assisted deposition
}

\author{
Li-Jian Meng ${ }^{\text {a,b,*}}$, Jinsong Gao ${ }^{c}$, M.P. dos Santos ${ }^{\mathrm{d}}$, Xiaoyi Wang ${ }^{\mathrm{c}}$, Tongtong Wang ${ }^{\mathrm{c}}$ \\ a Departamento de Física, Instituto Superior de Engenharia do Porto, Rua Dr. António Bernardino de Almeida, 431, 4200-072 Porto, Portugal \\ ${ }^{\mathrm{b}}$ Centro de Física, Universidade do Minho, 4700 Braga, Portugal \\ c Center of Optical Technology, Changchun Institute of Optics, Fine Mechanics and Physics of Chinese Academy of Science, PO Box 1024, 16\# East Nanhu Road, \\ Changchun 130033, China \\ d CeFITec, Universidade Nova de Lisboa/Departamento de Física, Universidade de Évora, Portugal
}

Received 4 May 2006; received in revised form 10 January 2007

Available online 5 May 2007

\begin{abstract}
Indium tin oxide (ITO) thin films have been deposited onto polycarbonate substrates by ion beam assisted deposition technique at room temperature. The structural, optical and electrical properties of the films have been characterized by X-ray diffraction, atomic force microscopy, optical transmittance, ellipsometric and Hall effect measurements. The effect of the ion beam energy on the properties of the films has been studied. The optical parameters have been obtained by fitting the ellipsometric spectra. It has been found that high quality ITO film (low electrical resistivity and high optical transmittance) can be obtained at low ion beam energy. In addition, the ITO film prepared at low ion beam energy gives a high reflectance in IR region that is useful for some electromagnetic wave shielding applications.
\end{abstract}

(C) 2007 Elsevier B.V. All rights reserved.

Keywords: ITO; Thin film; Polycarbonate; Ion beam assisted deposition

\section{Introduction}

Indium tin oxide (ITO) is an n-type degenerate wide band gap semiconductor. The degeneracy is caused by both oxygen vacancy and substitutional tin created during deposition. ITO thin films are widely used in optoelectronic devices such as solar cells, electroluminescence and liquid crystal displays as transparent electrode due to its high transmittance in the visible region and low electrical resistivity [1-3]. The high electrical conductivity of ITO films results in a high reflectivity in the infrared region, which gives applications to thermal insulation of windows, prevention of radiative cooling, etc. Many techniques have been used to deposit ITO films, such as sputtering, evaporation, sol-gel, etc. [4-6]. In general, ITO films deposited by the techniques mentioned above involve a

\footnotetext{
* Corresponding author. Departamento de Física, Instituto Superior de Engenharia do Porto, Rua Dr. António Bernardino de Almeida, 431, 4200072 Porto, Portugal. Tel.: +351 962325429; fax: +351 228321159 .

E-mail address:1jm@isep.ipp.pt (L.-J. Meng).
}

relatively high substrate temperature (over $200{ }^{\circ} \mathrm{C}$ ) or a postannealing process to obtain a reasonably high electrical conductivity and optical transmittance in the visible region $[7,8]$. Recently, plastic materials are desirable in the flat panel display industry, where the demand for remote information access is driving the development of rugged, lightweight, power efficient displays. These applications need to deposit ITO thin films onto plastic substrate. However, it is necessary for ITO thin films to be deposited at a very low substrate temperature because of the poor thermal endurance of plastic substrate. That means some traditional techniques we mentioned above are not suitable to deposit ITO films onto polymer substrate. Therefore, one of the important technical issues in the manufacturing processes of flexible optoelectronic devices is to develop a low temperature deposition technique. Ion beam assisted deposition (IBAD) has been considered as a low temperature deposition technique and has been used to deposited ITO films $[9,10]$. By this technique, colliding auxiliary gas ions can increase the adatom mobility; in addition, its energetic collision increases the substrate temperature of the very local colliding point by 
Table 1

Properties of ITO films on PC substrates

\begin{tabular}{lrrrr}
\hline Sample & PC1 & PC2 & PC3 & PC4 \\
\hline Screen voltage $(\mathrm{V})$ & 600 & 500 & 400 & 300 \\
Thickness $(\mathrm{nm})$ & 227 & 217 & 236 & 230 \\
Sheet resistance $(\Omega /$ square $)$ & 60 & 43 & 30 & 27 \\
Resistivity $\left(\times 10^{-3} \Omega \mathrm{cm}\right)$ & 6.2 & 4.2 & 3.2 & 2.8 \\
Carrier concentration $\left(\times 10^{20} \mathrm{~cm}^{-3}\right)$ & 3.5 & 3.1 & 4.7 & 3.1 \\
Hall mobility $\left(\mathrm{cm}^{2} / \mathrm{V}^{*} \mathrm{~s}\right)$ & 13.1 & 21.3 & 18.6 & 31.8 \\
Surface roughness by AFM $(\mathrm{nm})$ & 1.6 & 1.4 & 1.4 & 1.2 \\
Measured IR reflectance at $10,000 \mathrm{~nm}$ & $44 \%$ & $52 \%$ & $62 \%$ & $70 \%$ \\
Calculated IR reflectance & $36 \%$ & $54 \%$ & $68 \%$ & $71 \%$
\end{tabular}

momentum transfer [11]. Therefore, a high optical transmittance in the visible region and a low electrical resistivity of ITO films can be obtained at a much lower temperature than that of the conventional deposition techniques [12,17].

Although some jobs have been done on ITO films deposited onto polycarbonate (PC) substrates by various techniques $[14,18-20]$, it is hardly to find a detailed work on ITO films deposited onto PC substrates by ion beam assisted deposition method, especially the effect of the deposition parameters on film's properties. In this work, we have deposited ITO films onto PC substrates by using ion beam assisted deposition technique. The effect of the ion beam energy on the properties of the ITO films has been discussed.

\section{Experimental procedures}

ITO films were deposited onto the commercial polycarbonate substrates at room temperature by ion beam assisted deposition technique using a vacuum coater equipped with electron beam gun and a Kaufman ion source. ITO powder pellet with a composition of $90 \mathrm{wt} . \% \mathrm{In}_{2} \mathrm{O}_{3}$ and $10 \mathrm{wt} . \% \mathrm{SnO}_{2}$ was used as the evaporation source material. A $120-\mathrm{mm}-$ diameter Kaufman ion source was used to generate oxygen ion beam. The oxygen gas flow was controlled by a mass flow controller and was kept at $40 \mathrm{sccm}$ for all depositions. The deposition rate and the film thickness were monitored and controlled by a quartz crystal sensor which has been linked to ebeam power supply for automatic controlling. The nominal deposition rate and the thickness were preset at $0.2 \mathrm{~nm} / \mathrm{s}$ and $200 \mathrm{~nm}$, respectively. The substrate holder was rotated at a speed of 0.3 rounds $/ \mathrm{s}$. The angle between the incident oxygen ion beam and the normal of the substrate holder was fixed at $45^{\circ}$. Before the deposition, the chamber was evacuated until a pressure of $1 \times 10^{-3} \mathrm{~Pa}$. After that, the oxygen gas was introduced into the chamber. During the deposition, the dynamic pressure in the chamber was about $2.3 \times 10^{-2} \mathrm{~Pa}$. The film thickness indicated in the Table 1 was obtained by fitting the ellipsometric spectra. During the all depositions, the ion beam current and the accelerating voltage were kept constant at $100 \mathrm{~mA}$ and $250 \mathrm{~V}$, respectively. The screen voltage was varied from $300 \mathrm{~V}$ to $600 \mathrm{~V}$. Although we did not measure the ion beam energy, the screen voltage is directly related with ion beam energy. The higher screen voltage produces higher ion beam energy, so it is possible to study the ion beam energy effect by monitoring the variation of the screen voltage.

The optical transmittance spectra of the films were recorded by Perklin-Elmer Lambda 900 UV/VIS/NIR spectrometer and the infrared reflectance was tested by Perklin-Elmer Spectrum GX. The ellipsometric parameters Psi and Delta were measured using a Jobin-Yvon UVISEL ellipsometer. The ellipsometric Psi and Delta data were acquired at $70.25^{\circ}$ of incidence angle over the spectral range $300-800 \mathrm{~nm}$ in steps of $5 \mathrm{~nm}$. Atomic force microscopy (AFM) measurements were made using equipment from Digital Instruments Veeco Metrology Group. The X-ray diffraction was done by SHIMADZU XRD-6000 performed between the $2 \theta$ values of $20^{\circ}-60^{\circ}$ with a step of $0.05^{\circ}$. The Hall effect was measured using Lake Shore 665 with a $5-\mathrm{kG}$ magnetic field intensity at room temperature.

\section{Results and discussion}

Fig. 1 shows the X-ray diffraction spectra of the ITO films deposited at different screen voltages. It can be seen that the film deposited at the screen voltage lower than $400 \mathrm{~V}$ has an amorphous structure. When the screen voltage is higher than $500 \mathrm{~V}$, the film shows polycrystalline structure with a very small diffraction peak corresponding to (222) of $\mathrm{In}_{2} \mathrm{O}_{3}$ reflection. When energetic ions collide with the substrate, their kinetic energies are released either as heat energy at localized points of the substrate or as increased surface adatom mobility [13]. As the screen voltage is increased, the ion beam energy increases too. Thus, it is supposed that the additional heat energy and increased surface mobility of adatom by ion bombardment result in polycrystalline ITO growth at high screen voltage.

The surface morphology of the ITO films deposited at different screen voltages was studied by AFM measurements as shown in Fig. 2. It can be seen that the film prepared at high

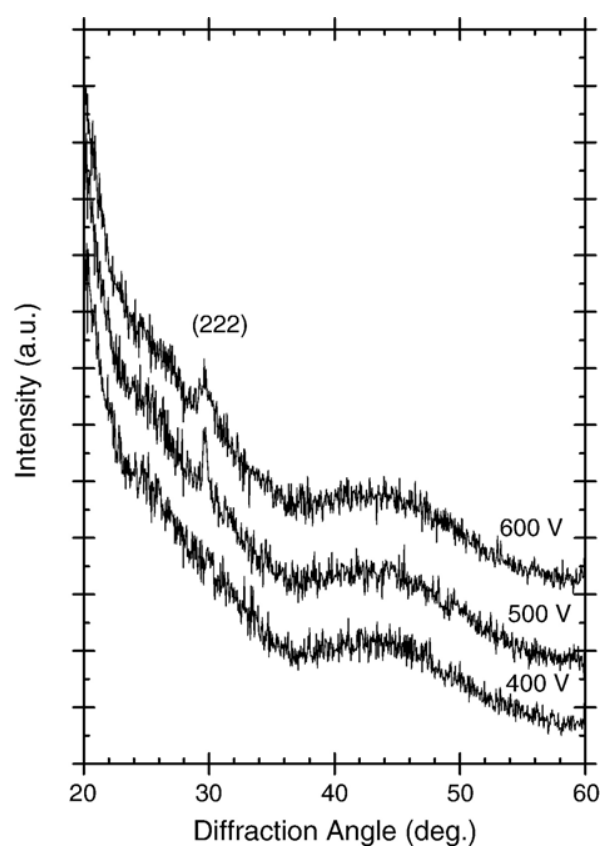

Fig. 1. XRD spectra of the ITO films deposited at different screen voltage. 


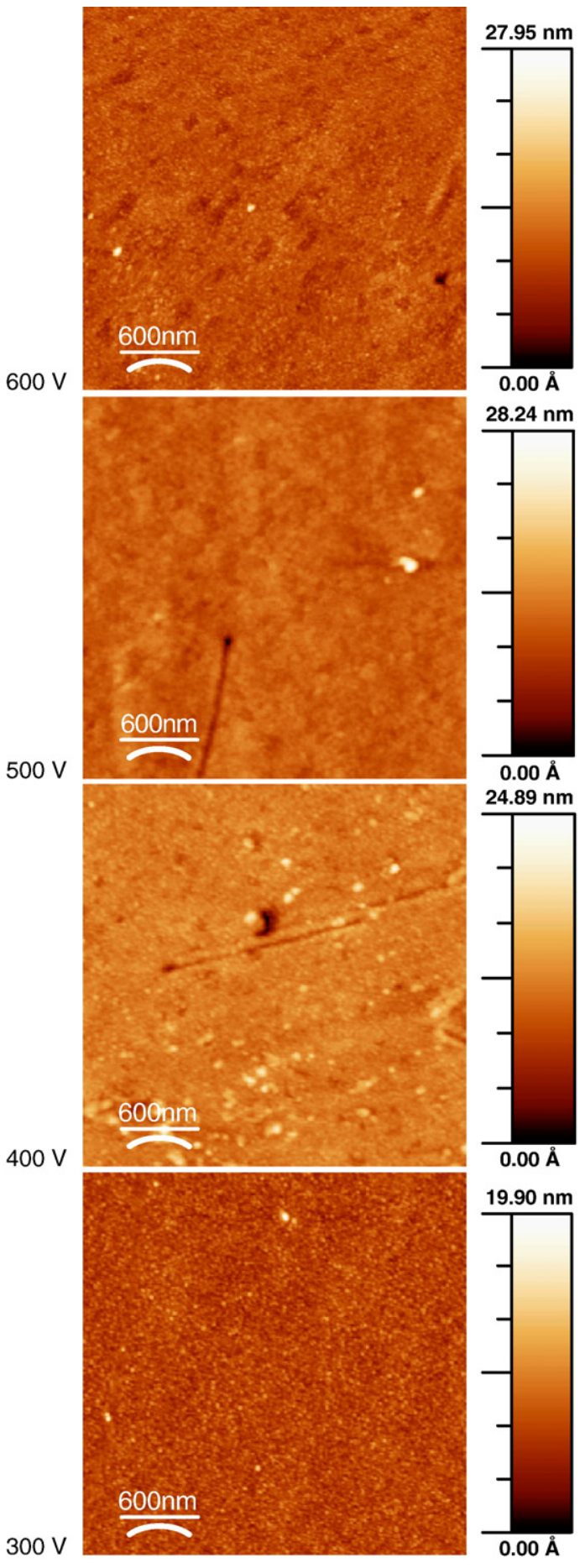

Fig. 2. AFM images $(3 \mu \mathrm{m} \times 3 \mu \mathrm{m})$ of the surface of the ITO films deposited at different screen voltages.

screen voltage shows some small pits in the surface and the film deposited at low screen voltage shows a smooth surface. The root mean square (RMS) surface roughness of the ITO films deposited at different screen voltages has been given in Table 1. It can be seen that the rms has a small increase as the screen voltage is increased. The similar result has been reported by Kim [14] for ITO films deposited by direct metal ion beam deposition. The bombardment of the substrate by high energy ion beam may result in this increase of the rms surface roughness.

Fig. 3 shows the specular transmittance spectra of the ITO films deposited at different screen voltages. A very clear decrease of the transmittance can be observed as the screen voltage is increased. It has be known that the surface roughness increases as the screen voltage is increased as shown in Fig. 2 and will result in a decrease of the transmittance because of the light scattering by the rough surface. However, this small variation in the surface roughness can not give a so big influence on the transmittance. Therefore, it must have the other reason for this decrease of the transmittance. The XRD spectra show that the film prepared at low screen voltage has an amorphous structure and the film prepared at high screen voltage has a polycrystalline structure. The amorphous phase may result in less light scattering and give a high transmittance. In addition, the film prepared at high screen voltage may have a volume inhomogeneity and result in an increasing of light scattering and give a low transmittance.

In order to get the optical constants (refractive index $n$ and extinction coefficient $k$ ) and the film thickness of the ITO films deposited at different screen voltages, we have made the ellipsometric measurements for all samples. The ellipsometric spectra have been fitted using the Lorentz oscillator model (classical model). The dielectric function in the visible region for classical model can be described as follows:

$\varepsilon(\omega)=\varepsilon_{\infty}+\frac{\left(\varepsilon_{\mathrm{S}}-\varepsilon_{\infty}\right) \omega_{t}^{2}}{\omega_{t}^{2}-\omega^{2}+i \Gamma_{0} \omega}$

where $\varepsilon_{\mathrm{S}}$ is static dielectric constant, $\varepsilon_{\infty}$ is high-frequency dielectric constant, $\omega_{t}$ is the characteristic frequency of the main oscillator and $\Gamma_{0}$ is the damping factor.

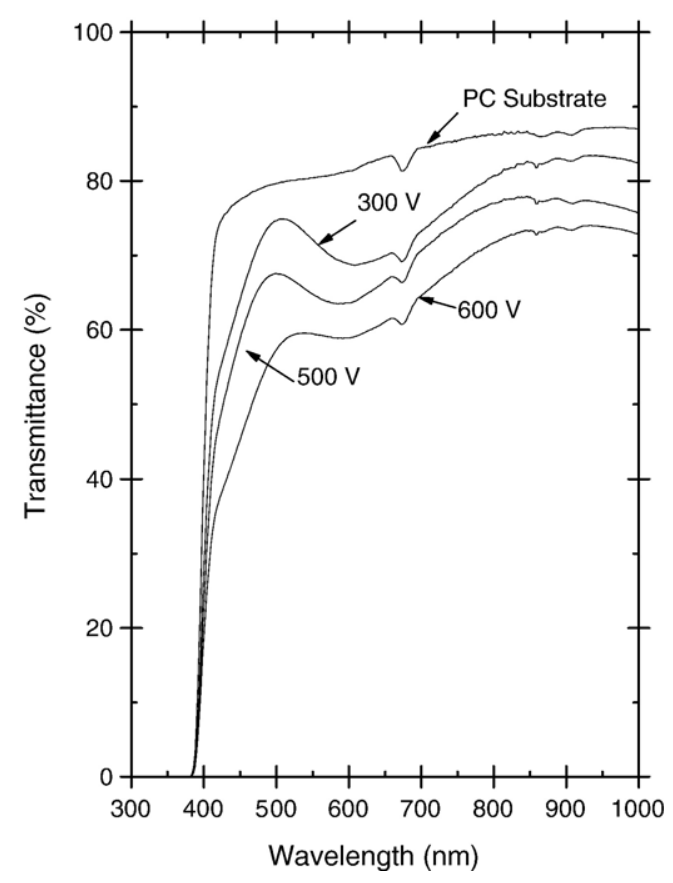

Fig. 3. Specular transmittance as a function of the wavelength for the ITO films deposited at different screen voltages. 
The dispersions of the refractive index and the extinction coefficient can be extracted from the fittings. The results are shown in Fig. 4. It can be seen from this figure that the film prepared at high screen voltage has a high refractive index and also a high extinction coefficient. The increase of the value of refractive index $n$ with screen voltage indicates that ion bombardment during deposition modifies the growth of film microstructure resulting in film densification. However, the refractive index of the film has a big variation when the screen voltage increases from $300 \mathrm{~V}$ to $500 \mathrm{~V}$. It can be related to the variation of the film structure. From Fig. 2 it can be seen that the film prepared at $300 \mathrm{~V}$ screen voltage shows an amorphous structure and the film prepared at screen voltage higher than $500 \mathrm{~V}$ shows a polycrystalline structure. The polycrystalline structure may have big refractive index than an amorphous structure has and result in this variation of the refractive index. The variation of the extinction coefficient can be related to the film optical transmittance as shown in Fig. 3, low transmittance gives a high extinction coefficient.

The electrical properties of ITO films prepared at different screen voltages have been given in Table 1 and shown in Fig. 5. Fig. 5a gives the variations of sheet resistance and electrical resistivity as a function of the screen voltage. The ITO film prepared at low screen voltage shows the low electrical resistivity. Fig. $5 \mathrm{~b}$ gives the variations of carrier concentration and mobility as a function of the screen voltage. Although the carrier concentration does not show a clear variation with the screen voltage, the carrier mobility does show a clear decrease as the screen voltage is increased. This decrease of the Hall mobility results in the increase of the electrical resistivity of the ITO films as the screen voltage is increased. The rough surface will be the one reason for the decrease of the Hall mobility.

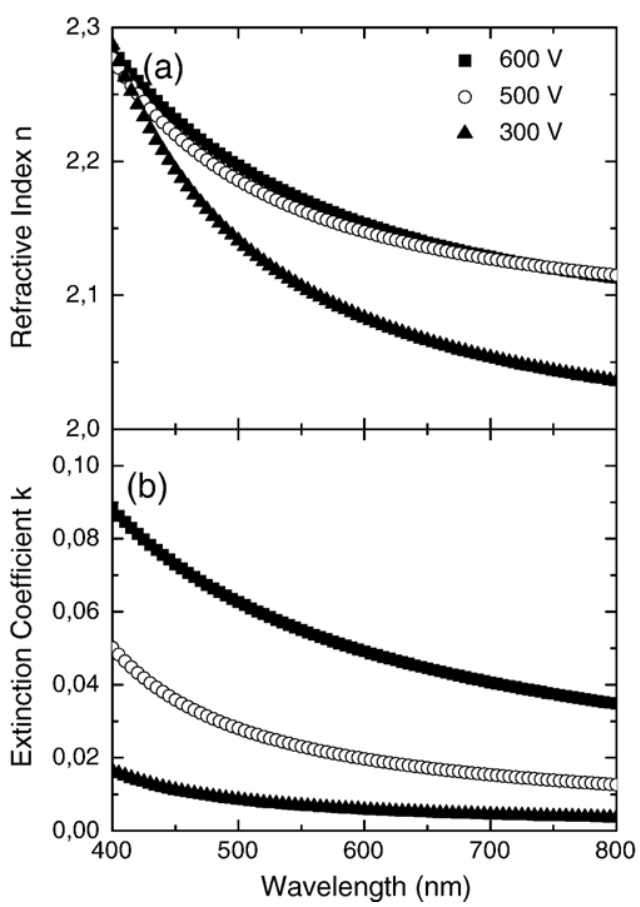

Fig. 4. Refractive index (a) and extinction coefficient (b) as a function of the wavelength for the ITO films deposited at different screen voltages.

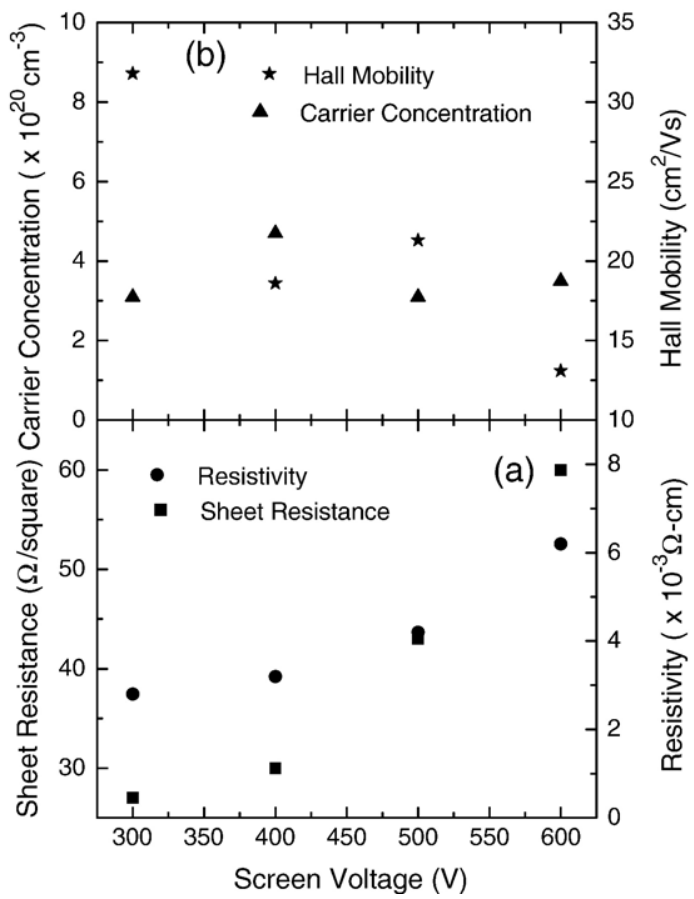

Fig. 5. Sheet resistance and resistivity (a) and carrier concentration and Hall mobility (b) of ITO films deposited at different screen voltages.

From Table 1, it can be seen that the surface roughness increases as the screen voltage is increased and will result in a decrease of the Hall mobility. In addition, the ionized impurity scattering, the neutral defect scattering and the negatively charged oxygen scattering may also contribute this decrease of the Hall mobility [15].

The infrared reflectance spectra of ITO films deposited at different screen voltages are given in Fig. 6. It can be seen that

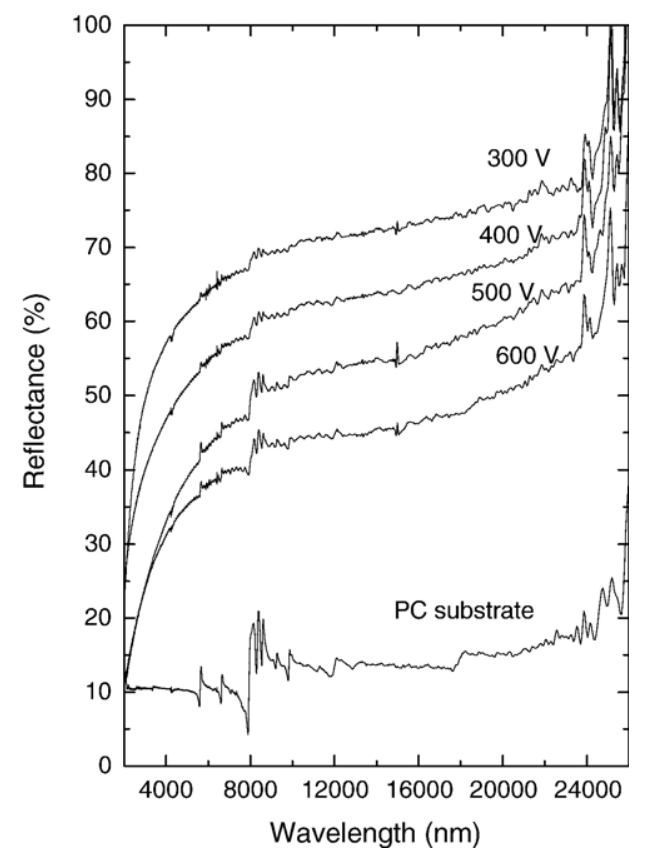

Fig. 6. Infrared reflectance spectra for ITO films deposited at different screen voltages. 
the reflectance increases as the screen voltage is decreased. By using the classical dispersion relations for the complex dielectric function of a free electron gas, Frank et. al. [16] showed that the infrared reflectance $R$ for high reflection level can be expressed by

$R=1-\frac{4 \varepsilon_{0} c_{0}}{e} \frac{1}{d N \mu}$

where $\varepsilon_{0}($ thepermittivity ofelectroninvacuum $)=8.85 \times 10^{-12}, c_{0}$ (velocity of light in vacuum $)=3 \times 10^{8} \mathrm{~m} / \mathrm{s}, e($ electronic charge $)=$ $1.6 \times 10^{-19} \mathrm{C}, N$ is the carrier concentration, $d$ is the film thickness and $\mu$ is the carrier mobility.

We have calculated the infrared reflectance of ITO films prepared at different screen voltages. The results are given in Table 1. For comparing, the measured infrared reflectance at $10000 \mathrm{~nm}$ is also given in Table 1 . It can be seen that for the film prepared at low screen voltage, the calculated reflectance is quite similar to the measured value.

\section{Conclusions}

ITO films have been deposited onto polycarbonate substrate at room temperature by ion beam assisted deposition technique at different screen voltages. The film prepared at low screen voltage shows an amorphous structure and the film prepared at high screen voltage shows a polycrystalline structure. The film surface roughness increases as the screen voltage is increased. The film prepared at low screen voltage gives a high optical transmittance, a low extinction coefficient and a low refractive index. The electrical resistivity of the film increases as the screen voltage is increased. Although the carrier concentration does not show a clear dependence on the screen voltage, the film prepared at low screen voltage has high carrier mobility. The infrared reflectance of the ITO films shows a clear increase as the screen voltage is decreased. The infrared reflectance has been calculated by theoretical model and it gives similar results comparing to the experimental values.

\section{Acknowledgments}

This work was carried out in the Centre of Optical Technology, Changchun Institute of Optics, fine Mechanics and Physics of Chinese Academy of Sciences during Li-Jian Meng's visit to the centre as a senior visiting scholar. Li-Jian Meng is thankful to the Fundação Calouste Gulbenkian for providing a fellowship.

\section{References}

[1] I. Hamberg, C.G. Granqvist, J. Appl. Phys. 60 (1986) R123.

[2] K.L. Chopra, S. Major, D.K. Pandya, Thin Solid Films 102 (1983) 1.

[3] C.G. Granqvist, A. Hultaker, Thin Solid Films 411 (2002) 1.

[4] Y.S. Jung, Solid State Commun. 129 (2004) 491.

[5] A.S.A.C. Diniz, C.J. Kiely, Renew. Energy 29 (2004) 2037.

[6] P.K. Biswas, A. De, N.C. Pramanik, P.K. Chakraborty, K. Ortner, V. Hock, S. Korder, Mater. Lett. 57 (2003) 2326.

[7] L.J. Meng, M.P. dos Santos, Thin Solid Films 322 (1998) 56.

[8] L.J. Meng, F. Placido, Surf. Coat. Technol. 166 (2003) 44.

[9] C. Liu, T. Mihara, T. Matsutani, T. Asanuma, M. Kiuchi, Solid State Commun. 126 (2003) 509.

[10] M. Gilo, R. Dahan, N. Croitoru, Opt. Eng. 38 (1999) 953.

[11] Y.W. Ko, S.I. Kim, J. Vac. Sci. Technol., A 15 (1997) 2750.

[12] D. Kim, S. Kim, Surf. Coat. Technol. 154 (2002) 204.

[13] K. Ogata, Y. Andoh, Nucl. Instrum. Methods B 80/81 (1993) 1427.

[14] D. Kim, Appl. Surf. Sci. 218 (2003) 70.

[15] H.C. Lee, O.O. Park, Vacuum 77 (2004) 69.

[16] G. Frank, E. Kauer, H. Kostlin, Thin Solid Films 77 (1981) 107.

[17] Y. Yang, Q. Huang, A.W. Metz, J. Ni, S. Jin, T.J. Marks, M.E. Madsen, A. DiVenere, S.-T. Ho, Adv. Mater. 16 (2004) 321.

[18] W.F. Wu, B.S. Chiou, Thin Solid Films 298 (1997) 221.

[19] D. Kim, S. Kim, Surf. Coat. Technol. 176 (2003) 23.

[20] T.K. Yong, T.Y. Tou, B.S. Teo, Appl. Surf. Sci. 248 (2005) 388. 